\title{
Cryo Focused Ion Beam Applications in High Resolution Electron Microscopy Studies of Beam Sensitive Crystals
}

\author{
Daliang Zhang ${ }^{1 *}$, Nini Wei ${ }^{1}$, Lingmei Liu ${ }^{2}$, Kepeng Song ${ }^{2}$, Ali Behzad ${ }^{1}$, Alessandro Genovese ${ }^{1}$, Yu \\ $\mathrm{Han}^{2}$ \\ 1. King Abdullah University of Science and Technology (KAUST), Core Labs, Thuwal, Saudi Arabia \\ 2. KAUST, Advanced Membranes and Porous Materials Center, Physical Sciences and Engineering \\ Division, Thuwal, Saudi Arabia \\ * Corresponding author: daliang.zhang@kaust.edu.sa
}

Imaging of electron beam sensitive materials is one of the most difficult applications of High-resolution Transmission Electron Microscopy (TEM). The challenges include the TEM sample preparation, the acquisition of images with extremely low beam doses, the time-constrained search for crystal zone axes, the precise image alignment, and the accurate determination of the defocus value. Encouraged by the success of atomic resolution TEM study on ZIF-8 Metal Organic Frameworks (MOF) by using the counting mode of K2 camera [1], we worked on a reliable methodology for HRTEM studies of electron beam sensitive crystals. A suite of methods have been developed to fulfill these requirements [2]. The images allow us to identify bulk structures, various types of surface termination and structure defects [3]. However, the application of these methods is limited to nano-sized crystals, as these crystals were so fragile that mechanical processes may cause structural damage.

Focused Ion Beam (FIB) provides us a promising way to prepare TEM samples from large sized specimens. But this technique is not easy to be used for radiation sensitive crystals due to the ion beam damage. Recently Cryo-FIB has been applied to prepare TEM lamellas for life science specimens. Therefore, we believe it is possible to develop experimentally a protocol to make thin lamella from electron beam sensitive crystals by Cryo-FIB.

MOF, Hybrid perovskite materials were used as exam samples. It is proved in our studies that beam damage of FIB can be controlled effectively in Cryo- conditions. Based on the crystal morphology, TEM lamella according to a specific preferred zone axis can be prepared, with large sized thin areas. The highest resolution observed in TEM images is about $0.14 \mathrm{~nm}$ from an HKUST MOF crystal. Structural dislocations, grain boundaries are observed by low dose HRTEM imaging with atomic resolution.

This work of preparing TEM specimens with Cryo- FIB, for MOF crystals and Hybrid perovskite materials, can substantially expanding the range of applications for low dose HRTEM imaging [4].

\section{References:}

[1] Y Zhu et al., Nature Materials 16 (2017), p. 532.

[2] D Zhang et al., Science, 6376 (2018), p. 675.

[3] L Liu et al., Nature Chemistry, 2019 (accepted)

[4] This research used resources of the Core Labs of King Abdullah University of Science and Technology (KAUST) 


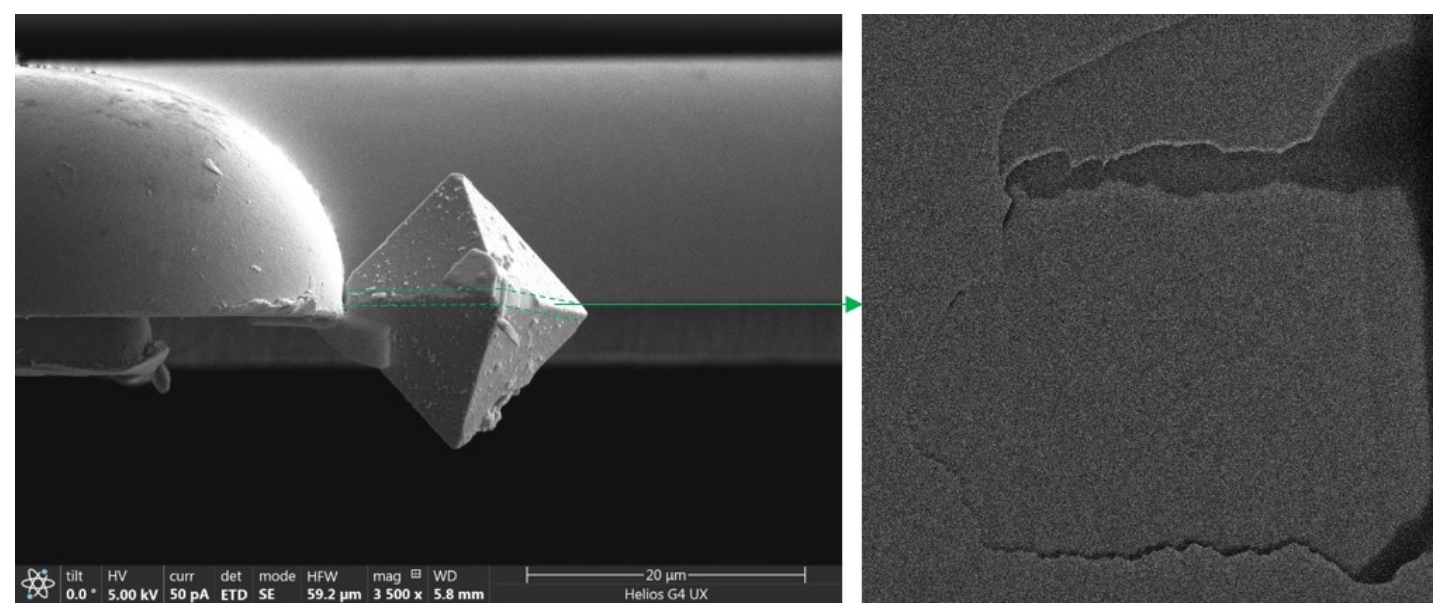

Figure 1. (left) SEM image of an orientated MOF crystal and (right) a TEM image of FIB lamella that prepared in Cryo- condition.
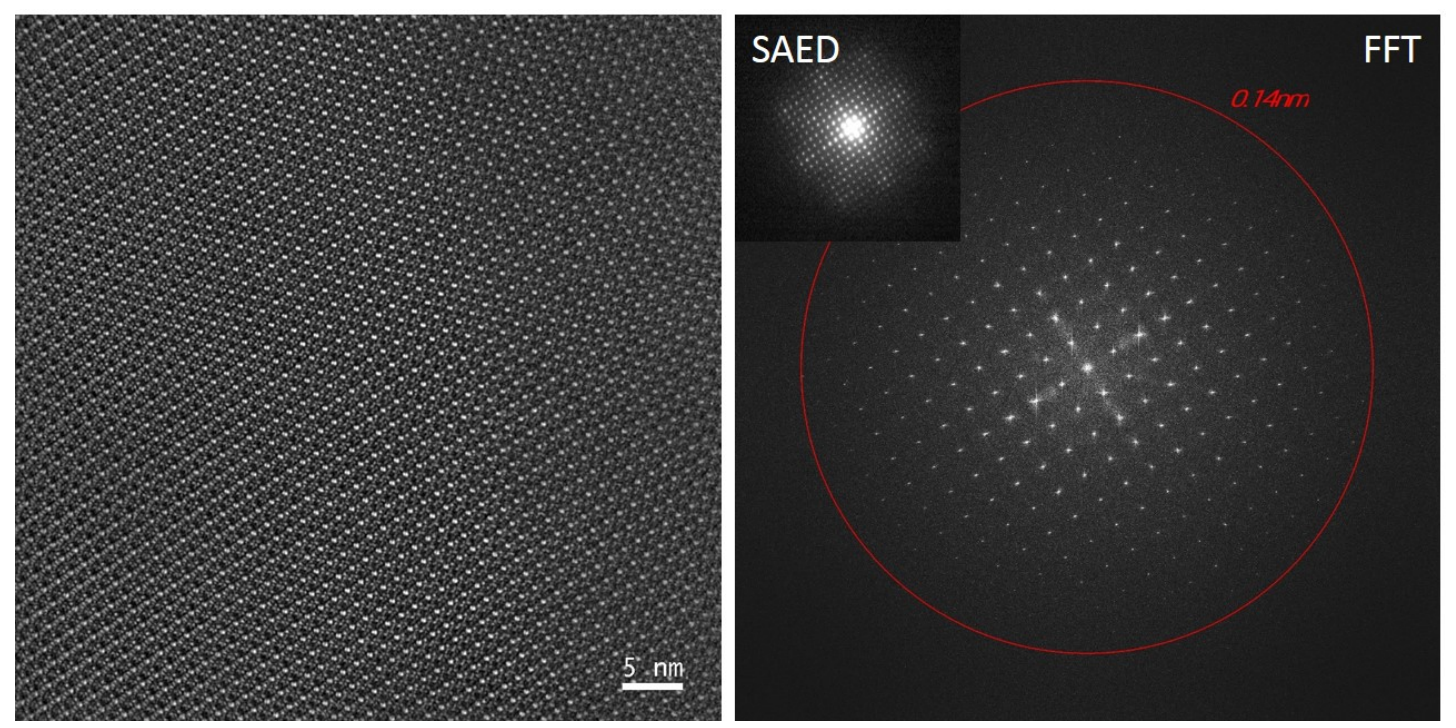

Figure 2. (left) HRTEM image taken from [001] zone axis of HKUST MOF crystal and (right) selected area electron diffraction, FFT patterns from the imaging area. Reflections around $0.14 \mathrm{~nm}$ d-spacing are well resolved. 